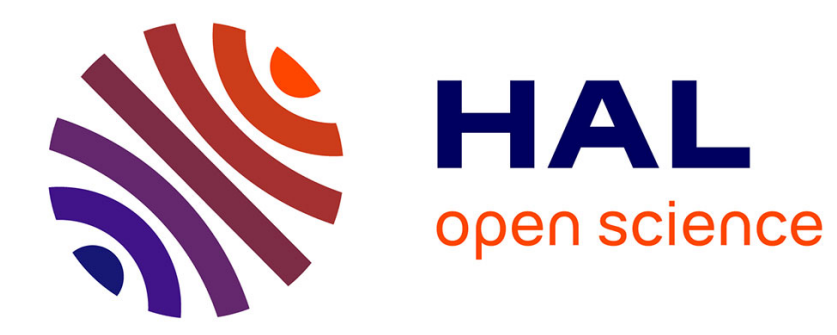

\title{
La compensation sociale du bonheur
}

Caroline Guibet Lafaye

\section{- To cite this version:}

Caroline Guibet Lafaye. La compensation sociale du bonheur. Le Philosophoire, 2006, 26, p. 99-112. hal-00372829

\section{HAL Id: hal-00372829 \\ https://hal.science/hal-00372829}

Submitted on 2 Apr 2009

HAL is a multi-disciplinary open access archive for the deposit and dissemination of scientific research documents, whether they are published or not. The documents may come from teaching and research institutions in France or abroad, or from public or private research centers.
L'archive ouverte pluridisciplinaire HAL, est destinée au dépôt et à la diffusion de documents scientifiques de niveau recherche, publiés ou non, émanant des établissements d'enseignement et de recherche français ou étrangers, des laboratoires publics ou privés. 


\title{
LA COMPENSATION SOCIALE DU BONHEUR ${ }^{1}$
}

\author{
Caroline Guibet LAFAYE
}

\author{
Centre Maurice Halbwachs \\ CNRS \\ 48, bd Jourdan, F-75014 Paris (France) \\ caroline.guibet-lafaye@ens.fr
}

\begin{abstract}
Résumé : Durant les années 1980-90, l'économie normative a délaissé la perspective du bien-être pour se concentrer sur les ressources et les chances. Pourtant, on trouve aujourd'hui un regain d'intérêt pour le bien-être. C'est donc aux usages post-welfaristes du bien-être, et en particulier à l'usage des functionings, que nous voudrions consacrer cette notre étude.
\end{abstract}

«Le plus grand bonheur du plus grand nombre est la mesure du juste et de l'injuste »telle est la matrice fondamentale de l'utilitarisme, dont J. Bentham prononce la formule au début du Fragment sur le gouvernement. Le bonheur, le bien-être entendu au sens du welfare, devient alors la mesure de la valeur et l'utilité, conçue de façon élargie comme relative à tout ce qui procure une satisfaction sans être nécessairement «utile», constitue un concept central $^{2}$. Toutefois le bonheur dont l'utilitarisme fait le critère de la moralité est avant tout collectif et consiste dans la somme des niveaux de bien-être (ou d'utilité) des individus, qui composent la collectivité considérée. S'il est vrai que, dans cette perspective, seule importe la somme du bien-être et non la manière dont elle est distribuée, en revanche la distribution de parts équitables - notamment des revenus - entre les membres de la société importe au premier chef, parce que la maximisation de la somme du bien-être exige que le gain marginal en bien-être, dans l'affectation des ressources aux différents individus, soit partout le même. Cette tradition utilitariste est à l'origine de ce que l'on nomme, depuis le début du XXe siècle, l'économie du bien-être (welfare economics). Le welfarisme considère que la satisfaction subjective ou le bien-être - que l'on mesure à l'aide de la relation de préférence individuelle ou de la fonction d'utilité correspondante - représente la donnée principale, voire exclusive, de toute appréciation normative des règles collectives. L'économie du bien-être consiste alors à évaluer les situations économiques et, principalement, les modalités de la répartition, l'objectif étant l'identification d'un optimum.

Que l'on considère l'utilitarisme comme tel ou l'économie du bien-être, l'une des questions décisives est de déterminer non seulement la nature de la distribution mais aussi qui sont les individus dont l'utilité et le bien-être doivent être pris en compte. Ainsi certaines théories de la justice contemporaines considèrent que le bien-être doit faire l'objet d'une

\footnotetext{
${ }^{1}$ Article publié in Le Philosophoire, numéro sur le Bonheur, n²6, Printemps-Eté 2006, Paris, p. 99-112. [ISSN : 1283-7091]

${ }^{2}$ Plus précisément, l'utilité est cette « propriété que détient quelque objet que ce soit de tendre à produire un bénéfice, un avantage, du plaisir, le bien, ou le bonheur » (Bentham (1998), Introduction, I, 3).
} 
redistribution visant à compenser les inégalités interindividuelles. Telles sont donc les questions que nous voudrions explorer : comment le bonheur et son déficit peuvent-ils être socialement compensés? Peut-on concevoir des dispositifs dont l'effet redistributif serait d'induire davantage d'égalité en matière de bonheur? Afin d'élucider ces interrogations, il importe, en premier lieu, d'envisager le regain d'intérêt actuel pour les comparaisons interpersonnelles de bien-être, puis de déterminer dans quelle mesure les agents sont responsables de leur bonheur, pour enfin voir comment ce dernier peut être pris en compte comme un élément pertinent d'une redistribution des biens sociaux.

\section{1- Comparer le bien-être.}

\section{1-1 Le welfarisme et ses limites.}

Le welfarisme confère à l'égalité du bien-être une importance centrale et vise une maximisation du bien-être minimal. Or l'économie du bien-être, qui cherche à compenser les individus pour des niveaux d'utilité inégaux, se heurte à la difficile exigence de la comparabilité interpersonnelle du bien-être, puisque pour proposer une compensation des différences individuelles en matière d'utilité, il est requis de comparer les niveaux d'utilité des agents entre eux. Or Vilfredo Pareto et ses disciples ont montré que la satisfaction ne peut se comparer d'un individu à un autre'

De façon générale, le welfarisme est confronté à deux problèmes majeurs : le problème dit des «goûts dispendieux » et celui de «l'épouse complaisante». Le problème des "goûts dispendieux » désigne cette situation où un individu peut délibérément cultiver des "goûts dispendieux » et ensuite afficher des revendications pour des parts distributives plus importantes. Celui de «l'épouse complaisante», en revanche, souligne qu'un individu peut être opprimé de manières qui le conduisent à avoir une compréhension excessivement modeste de son bien-être ainsi que des revendications insignifiantes. Dans les deux cas, il est manifeste que l'on ne peut se satisfaire d'une évaluation de la part distributive de chacun à partir de la perception, par l'agent, de son bien-être ${ }^{2}$. En raison de difficultés de cette nature, le welfarisme a été rejeté en faveur d'approches plus objectives telles que l'égalitarisme ressourciste ${ }^{3}$.

En effet l'économie normative contemporaine s'est globalement dégagée du welfarisme, voire y est hostile. Elle stigmatise la conception du choix social des années 1970. Cette attitude est caractéristique des années 1980-1990 et se retrouve chez des auteurs aussi différents que Rawls, Dworkin, Sen, Cohen ou Roemer. La polémique s'est développée notamment - mais pas exclusivement - à l'occasion du débat lancé par Sen en 1980 posant la question: «Égalité de quoi?». Les critiques alors formulées ont conduit les théories égalitaristes de la justice à privilégier l'égalisation des ressources, personnelles et impersonnelles, y trouvant une solution aux problèmes rencontrés par le welfarisme. Elles s'attachent, selon les approches, à égaliser les ressources externes des agents, leurs dotations internes, leurs chances ou les résultats auxquels ils parviennent, et contribuent ainsi à expliciter l'information ne concernant pas l'utilité dans les jugements sur le bien-être. Ce courant «post-bien-êtriste» envisage, de façon privilégiée, les avantages objectifs, identifiables dans les situations individuelles, tels que la possession de "biens premiers ", comme le suggère par exemple J. Rawls dans sa Théorie de la justice de 1971.

\footnotetext{
${ }^{1}$ Voir W. Pareto, Manuel d'économie politique, 1906.

${ }^{2}$ Voir Keller (2002), pour une défense récente de la position welfariste, en particulier contre l'objection des « goûts dispendieux ». Keller montre qu'un problème similaire apparaît également au sein de la perspective ressourciste.

${ }^{3}$ Rawls (1971, 1982), Dworkin (2000), Van Parijs (1995).
} 


\section{1-2 La réhabilitation des comparaisons interpersonnelles d'utilité.}

Pourtant contre cette critique du welfarisme, A. Sen a montré qu'une réhabilitation fructueuse des comparaisons interpersonnelles d'utilité était possible. Les auteurs qui ont participé à ce regain d'intérêt - Sen lui-même dans Collective Choice and Social Welfare, d'Aspremont, Gevers, Hammond, Roberts, Blackorby notamment - ont dépassé l'impossibilité du théorème d'Arrow ${ }^{1}$. En particulier, l'orientation choisie par Sen consiste à souligner l'importance, dans l'interprétation et l'évaluation du bien-être, des réalisations (functionings) ou des capacités objectives (capabilities), parmi les caractéristiques individuelles qui ne peuvent être conceptuellement réduites aux fonctions d'utilité ${ }^{2}$. Sen recommande également d'inclure des caractéristiques plus subjectives dans l'évaluation des besoins et du bien-être, dans la mesure où les théories égalitaristes ressourcistes, qui se donnaient comme un dépassement du welfarisme ne peuvent totalement écarter ni délaisser le recours au bien-être. C'est en particulier ce que révèle l'analyse par J. Roemer de l'égalitarisme ressourciste de Dworkin.

\section{1-3 Egalisation des ressources et égalisation du bien-être.}

J. Roemer a, en effet, montré, dans l'article « Equality of Resources Implies Equality of Welfare $»^{3}$, qu'en fonction de la conception générale que l'on adopte de la ressource, l'égalisation des ressources et l'égalisation du bien-être sont indistinctes ${ }^{4}$. L'égalisation des ressources, dans une population dont les ressources sont attachées aux individus et ne peuvent, par conséquent, pas être transférées, ne peut pas simplement signifier, du fait de cette inaliénabilité physique, une égale division physique des ressources parmi cette population. Définir une égalité des ressources consiste, comme le montre Roemer, à déterminer un mécanisme, à partir d'environnements économiques donnés, vers un espace de distributions faisables sur ces environnements, puis à distribuer des ressources transférables, de telle sorte qu'elles compensent, de façon adéquate, les personnes pour leurs dotations non transférables. Une compensation est «appropriée » lorsque le mécanisme distributif satisfait plusieurs axiomes, élaborés en respectant un souci égalitariste. Ces axiomes constituent alors des conditions nécessaires pour une « égalisation des ressources » dans la population ${ }^{5}$.

Parmi les ressources qui peuvent être distribuées, certaines, comme les « talents », sont non transférables. Une perspective radicalement égalitariste motive une compensation pour des dotations différentes, en ressources non transférables - attachées aux personnes - dans la

\footnotetext{
${ }^{1}$ Arrow (1951).

${ }^{2}$ La notion de functioning, décrite par A. Sen, permet, par exemple, d'éviter le recours à la métrique contestable de l'utilité. Ainsi les indicateurs mesurant les capacités, loin de consacrer une approche strictement limitée aux «utilités » ou aux satisfactions psychologiques, tracent un pont vers la critique, menée par Sen, de l'usage exclusif, en éthique sociale, de l'information-utilité, c'est-à-dire du principe du welfarisme. En effet $\mathrm{A}$. Sen suggère que la vie peut être mise en équation par un ensemble de «functionings " (consistant en modes d'être et en faits) placés en corrélation, comme le fait d' "être nourri correctement», le fait d' " être heureux » (ou d' « éprouver du plaisir ») et le fait de « survivre». Chaque functioning désigne l'une des dimensions de ce qui peut importer dans la vie d'une personne : l'absence de maladie, le logement ou la participation économique et sociale, par exemple. L'état d'une personne est alors compris comme un vecteur de functionings et l'ensemble des vecteurs faisables constitue l'ensemble des capabilités (ou capacités). Or l'ensemble des capabilités fournit des informations sur la capacité d'une personne à réaliser son bien-être.

${ }^{3}$ Roemer (1986).

${ }^{4}$ Précisément, l'une des questions centrale de l'égalisation des ressources est la détermination de ce qu'une éthique égalitariste de la ressource suppose.

${ }^{5}$ Roemer (1986), p. 765.
} 
mesure où ces dotations sont moralement arbitraires. Or le seul mécanisme qui satisfasse ces axiomes, sur un large domaine de l'économie, est celui qui distribue les biens, de telle sorte qu'il égalise les utilités des agents ${ }^{1}$. Dès lors, l'unique mécanisme d'allocation satisfaisant certaines conditions minimales que l'égalitarisme de la ressource requiert tend à égaliser le bien-être des agents. Autrement dit l'examen des axiomes devant être satisfaits par n'importe quel mécanisme d'allocation égalitaire des ressources, dans un environnement économique donné, montre que ces axiomes exigent l'égalitarisme du bien-être. Il n'y a donc pas, comme le souligne Roemer, de distinction substantielle entre l'égalité des ressources et l'égalité du bien-être, quand les ressources sont comprises comme incluant des attributs internes des personnes $^{2}$. Si l'égalitarisme des ressources a un sens, les comparaisons interpersonnelles d'utilité doivent donc être admises ${ }^{3}$. Cette conclusion s'établit également $a$ contrario puisque si l'on supprime l'hypothèse de la comparabilité interpersonnelle d'utilité, il apparait qu'aucun mécanisme de distribution égalisant les ressources n'existe ${ }^{4}$. Le modèle de Dworkin, exclusivement tourné vers une distribution des ressources entre les agents et excluant la prise en compte du bien-être, appelle donc une correction, dans la mesure où tout en souscrivant à une forme radicale d'égalitarisme, ce modèle repose sur une distinction entre égalité des ressources et égalité du bien-être qui n'est pas tenable.

\section{2- Les individus sont-ils responsables de leur bonheur?}

Pas plus que l'approche welfariste, les théories ressourcistes ne permettent une compensation satisfaisante de l'état personnel final et par conséquent de l'état de bonheur ou de malheur d'un individu. L'un des paramètres majeurs qu'il est requis de considérer, en la matière, est le rôle joué par la responsabilité individuelle. En effet celle-ci est déterminante dans l'évaluation de la légitimité d'une revendication, formulée par un agent à l'égard de la communauté, en vue d'une compensation pour un état de bien-être donné. La question du rôle de la responsabilité personnelle, dans la détermination du bien-être individuel, se pose, depuis la Théorie de la justice de Rawls, dans les termes d'une division du travail entre les institutions sociales et les décisions personnelles, c'est-à-dire dans les termes d'une dissociation entre ce dont les individus sont responsables et ce dont la société est tenue pour responsable. De fait, l'accomplissement personnel, sur lequel repose le bonheur individuel, dépend de la façon dont l'agent, prudemment ou non, emploie ses ressources totales ${ }^{5}$.

Selon les modèles de justice distributive envisagés, le statut conféré à la responsabilité varie. Les théories de l'égalité des chances ${ }^{6}$ jugent que la société doit assurer aux individus des chances, ceux-ci étant responsables de les saisir et de les faire, par la suite, fructifier. Les théories de l'égalité des ressources, pour leur part, considèrent que la société doit fournir des ressources aux individus, ces derniers étant responsables de leur usage ainsi que de leurs ambitions. L'approche par les capabilités, quant à elle, vise à assurer l'accès, aux individus, à la plus large gamme de possibilités dans la mesure où, pour cette approche, la liberté constitue

\footnotetext{
${ }^{1}$ « The conclusion is that only one allocation mechanism passes the various tests required for implementing equality-of-resources, the mechanism that equalizes the welfares of the agents » (Roemer (1986), p. 766).

${ }^{2}$ Roemer (1986), p. 752.

3 Jan Tinbergen suggère également que l'utilité peut être mesurée et que, de ce fait, des comparaisons interpersonnelles peuvent être faites (Tinbergen (1957), p. 500).

${ }^{4}$ Voir Roemer (1986), VII.

${ }^{5}$ On suppose en effet qu'il est possible d'opérer une distinction entre la chance et la responsabilité dans l'espace des accomplissements aussi bien que dans le champ des caractéristiques individuelles. Les accomplissements ou les caractéristiques sont alors identifiables à des « biens ».

${ }^{6}$ Voir Sen (1985a, 1987, 1992), Arneson (1989, 1990), Cohen (1989, 1990), Roemer (1996), Vallentyne (2000).
} 
une dimension importante du bien-être ${ }^{1}$. Les théories égalitaristes du bien-être considèrent qu'une inégalité dans le bien-être est, en tant que telle, pertinente et la responsabilité personnelle y est directement prise en compte. Sont alors écartés, de toute compensation, les individus qui ont librement choisi de s'engager dans des activités ayant pour résultat un déficit du bien considéré, ce déficit leur étant alors légitimement attribué. De même, les théories indirectes de la justice comme celle de Rawls considèrent que les «variations de préférences et de goûts sont considérées comme relevant de notre seule responsabilité » ${ }^{2}$. La « division sociale des responsabilités » signifie alors que « la réussite ou l'échec, leur bonheur ou leur malheur sont des conséquences qui incombent [pour les individus] à leur propre responsabilité $\rangle^{3}$. Les individus sont tenus pour responsables de leurs actions. Ils en tirent les bénéfices et assument les coûts de leurs choix. La division sociale de la responsabilité a donc pour conséquence que certains désavantages demeurent de la responsabilité des individus. A l'inverse, on peut considérer que les individus ne sont responsables ni de leurs goûts ${ }^{4}$ ni de leurs préférences ${ }^{5}$. R. Arneson suggère, par exemple, que la société doit concevoir la redistribution des ressources et des biens, de telle sorte que les préférences, qui n'ont pas été choisies par les individus, ne les pénalisent pas dans la distribution de ces ressources.

Ces positions théoriques présentent néanmoins toutes des insuffisances. Alors que les théories de la justice welfaristes font «trop » dépendre la part distributive d'un individu de ses caractéristiques propres, les théories de la justice ressourcistes tiennent «trop peu» compte de telles caractéristiques ${ }^{6}$. En effet, les partisans du welfarisme nourrissent des revendications individuelles, pour des parts distributives, en fonction de facteurs qui influencent le bien-être, mais nombre de ces facteurs ne devraient pas être pris en compte, dans la distribution - comme l'indique le problème des goûts dispendieux. En revanche, les théories de la justice ressourcistes suggèrent que ce n'est pas la possession, comme telle, de ressources dont nous devons nous soucier - ou du moins pas exclusivement - mais de ce que ces ressources représentent pour les personnes ${ }^{7}$. Dans les deux approches, les principes welfaristes et les principes ressourcistes ne peuvent être mis en œuvre tels quels. Ils exigent des critères correctifs leur permettant de se concrétiser dans des mesures de justice redistributive. De même la méthode d'indifférence décrite par M. Fleurbaey et l'approche par les functionings, qui propose une pondération appropriée des déterminants des situations individuelles, offrent une solution en reconnaissant aux individus une pleine responsabilité pour leurs ambitions ou pour leur niveau d'utilité. Le bonheur est alors considéré comme un functioning approprié, au sujet duquel nos buts, dans l'existence, nous font exprimer des préférences et dont il convient de tenir compte dans l'appréhension du bien-être individuel ${ }^{8}$.

\footnotetext{
${ }^{1}$ Voir en particulier A. Sen $(1987,1992,1999)$.

${ }^{2}$ Rawls (2001), p. 228.

${ }^{3}$ Voir Scanlon (1998), p. 244.

${ }^{4}$ Certains agents peuvent avoir une aversion pour le travail salarié sans que cette aversion ne puisse être mise au compte de leur responsabilité puisqu'ils ne l'ont pas choisie.

${ }^{5}$ Nous verrons ultérieurement qu'il est possible de mettre en œuvre l'idée de responsabilité partielle pour les préférences et pour les goûts - conformément à la distinction faite par M. Fleurbaey entre les individus qui ont des goûts dispendieux mais qui, pour l'un, lorsqu'il en est privé n'est pas malheureux alors que l'autre l'est.

${ }^{6}$ Les difficultés, rencontrées par les approches welfaristes, pour intégrer des considérations relatives à la responsabilité, dans une théorie de la justice, sont manifestes dans les travaux d'Arneson. Sur les difficultés rencontrées par les égalitaristes ressourcistes, pour parvenir à une intégration similaire, voir en particulier Lippert-Rasmussen (2001) et Anderson (1999).

${ }^{7}$ Sans même considérer que ces ressources manquent, il apparaît que certaines personnes - en particulier les personnes handicapées - ont plus de besoins que l'agent qui en a déjà le plus, alors que d'autres personnes ont pu avoir agi de manières qui leur font mériter plus qu'une part « égale » des ressources.

${ }^{8}$ Rappelons la définition des functionings : «A functioning is an achievement of a person: what he or she manages to do or to be. It reflects, as it were, a part of the 'state' of that person. It has to be distinguished from the commodities which are used to achieve those functionings» (Sen (1985b), p. 7). Ainsi «être heureux et
} 


\section{3- Bonheur et accomplissements.}

\section{3-1 Résultats sociaux et accomplissements privés.}

En effet l'intérêt exclusif pour les ressources ou pour les chances, au détriment des accomplissements, des réalisations sociales fondamentales et des functionings, ne se justifie pas. A. Sen déjà considère que la liste des functionings appropriés ne doit se restreindre ni au bien-être subjectif - parce que certains accomplissements objectifs sont importants - ni aux ressources - parce que ce qui compte n'est pas tant ce que les gens ont que ce qu'ils peuvent réaliser avec leurs ressources ${ }^{1}$. Dès lors, certains functionings, issus d'une sélection d'accomplissements, que tout individu est et doit être en mesure de mener à bien, doivent être privilégiés. En particulier, il semble pertinent d'accorder une attention spécifique à l'épanouissement des fonctions humaines normales et au bien-être. Dans cette perspective, Marc Fleurbaey propose de s'intéresser, moins aux biens primaires ou aux ressources de départ, qu'aux réalisations fondamentales, auxquelles les individus peuvent parvenir, étant entendu que ces réalisations concernent les résultats jugés essentiels du point de vue de la condition sociale. Par là, ce sont les résultats, et non simplement les ressources, qui sont pris en considération quoique les résultats individuels, jugés d'intérêt purement privé - comme la satisfaction subjective -, soient négligés. Une pondération appropriée de ces dimensions consiste alors à attribuer à certains functionings un poids faible, voire nul, et à placer un poids substantiel sur les functionings qui traitent des dimensions de base de l'épanouissement humain. Cette définition restrictive des réalisations fondamentales dessine une sphère privée substantielle et répond à la division du travail, préconisée par Rawls, entre société et individu. En effet cette pondération, qui accorde une place privilégiée à l'épanouissement humain, trace une sphère de responsabilité, respectant la liberté des agents ${ }^{2}$, leurs préférences et leur choix de style de $v^{3} e^{3}$ sans, par ailleurs, compromettre leurs intérêts fondamentaux ni consolider le principe discutable d'inégalités méritocratiques ${ }^{4}$.

L'avantage de cette approche, par les résultats primaires ou réalisations fondamentales, est de prendre en compte le fait que les individus peuvent mal utiliser les ressources ou les chances, qui ont été mises à leur disposition, dans une situation fictive initiale. En effet, les théories de l'égalité des ressources aussi bien que les théories de l'égalité des chances n'excluent pas la possibilité - ni n'y remédient - que les individus puissent mal utiliser leurs ressources et leurs chances et finir dans des situations intolérables. Faute d'évaluer les situations individuelles en termes de bien-être réalisé, ces théories n'offrent aucun filet de sûreté, en matière d'accomplissement individuel. En revanche, lorsque les situations individuelles sont évaluées à partir d'une sélection d'accomplissements, identifiés comme des " résultats sociaux » ou comme des «functionings primaires » essentiels, pour le statut social individuel et les relations sociales, les individus ne peuvent être irrémédiablement condamnés

obtenir ce qu'on désire peuvent être évalués, entre autres, dans l'approche par les capabilités » (Sen (1993), p. 33).

${ }^{1}$ Sen montre en effet que l'avantage qui est retiré d'un bien n'est pas principalement du plaisir mais consiste plutôt dans l'opportunité de réaliser ses potentialités.

${ }^{2}$ Les capabilités expriment la dimension de la liberté alors que les functionings actuels témoignent de l'accomplissement auxquels les agents parviennent.

${ }^{3}$ Il est en effet essentiel que soit écartée l'objection selon laquelle l'approche retenue, puisqu'elle évalue les situations sur la base de caractères objectifs, tels que des functionings objectifs, s'écarte de l'évaluation par les individus de leur propre situation, dans la mesure où cela reviendrait à imposer une conception particulière de la vie bonne.

${ }^{4}$ Voir Fleurbaey (2004), p. 16-17. 
à des situations indignes ${ }^{1}$. Or une perspective égalitariste et solidariste présuppose qu'il n'est pas décent, pour les membres d'une communauté, de laisser d'autres individus vivre dans des conditions inférieures - ou médiocres -, quelle que soit la responsabilité morale qui leur incombe pour cette situation. Une certaine égalité sociale de base est ainsi garantie, alors même qu'une place est ménagée à la responsabilité individuelle, dans la mesure où cette pondération des functionings néglige certains accomplissements, relativement auxquels les individus ne peuvent recevoir d'aide, s'ils y manquent de succès.

\section{3-2 Pondérer les accomplissements.}

Comme il est apparu, l'évaluation des situations individuelles est confrontée, de façon paradigmatique, à la question de savoir quelle place accorder aux préférences individuelles. La pondération modulable des dimensions de l'épanouissement humain a montré qu'une personnalisation de l'index des functionings, respectant les préférences des individus, y compris à l'égard de ce dernier, est envisageable ${ }^{2}$. Précisément, la méthode d'indifférence permet de pondérer les préférences individuelles, la liberté et la responsabilité de façon distincte, par l'usage de courbes d'indifférence ${ }^{3}$. Le principe général de cette méthode consiste à décrire la situation d'un individu, non pas simplement par son vecteur de functionings, mais par sa courbe d'indifférence - au sein même de l'espace des functionings , laquelle contient son vecteur actuel de functionings. Un sentier de référence - relativement auquel n'importe quelle situation individuelle peut être évaluée - est construit, à partir de l'identification du vecteur du sentier de référence qui lui est équivalent, étant données les préférences de ce même individu, quant à sa situation actuelle. Par ce procédé, chaque individu est associé à un vecteur particulier du sentier de référence, que l'on désigne comme son «vecteur équivalent». L'ensemble des vecteurs équivalents étant rangé par ordre de domination, une comparaison des situations individuelles est possible de même qu'une identification des individus les plus mal lotis ou les plus défavorisés. La méthode d'indifférence respecte ainsi les préférences individuelles sans avoir recours à de problématiques comparaisons interpersonnelles de satisfaction subjective. Le sentier de référence, qu'il soit défini en termes de vecteurs ou en termes d'ensembles de budget, dessine alors l'ensemble des situations dans lesquelles les préférences individuelles peuvent être négligées. De façon générale, le respect des préférences individuelles exige de tenir compte de la forme des courbes d'indifférence mais aussi des préférences qui sont éloignées des situations individuelles courantes. Or cette méthode révèle que les préférences sont à placer, au moins en partie, hors de la sphère de la responsabilité. On démontre ainsi que les personnes peuvent ne pas être tenues pour entièrement responsables de leurs préférences et par conséquent de leur niveau d'utilité.

\footnotetext{
${ }^{1}$ On trouve une argumentation semblable dans Anderson (1999) et Hurley (2003). T. Scanlon (1975) a également proposé une version de ce type d'approche. Ajoutons que l'approche par « résultats sociaux » ne tisse pas seulement un filet de sûreté mais accorde également la priorité aux individus ayant les plus bas résultats dans les dimensions retenues, aussi élevés ces résultats soient-ils.

${ }^{2}$ Fleurbaey (2004), p. 17.

${ }^{3}$ Pour construire ces courbes d'indifférence, la seule information requise des individus est l'indifférence entre leur situation, pour chacun, et une situation de référence. Cette dernière est figurée par un sentier de référence, c'est-à-dire par un ensemble de points pour lesquels les situations individuelles sont comparables entre elles. Le sentier de référence est donc l'ensemble des points, qui sont équivalents, du point de vue de cette situation de référence ou situation d'équité.
} 


\section{3-3 Pondérer le bonheur, compenser les ambitions.}

L'approche par les functionings et la méthode d'indifférence ont donc le mérite d'isoler une pondération appropriée du bonheur, au sujet duquel les buts que nous poursuivons chaque jour nous font exprimer des préférences. Comme on le sait, il est difficile, dans un contexte multidimensionnel, de comparer des degrés différents de succès concernant des buts différents, pourtant la méthode d'indifférence permet de contourner cette difficulté, notamment dans une situation où le bonheur est l'un des functionings, au sujet duquel les individus ont des préférences.

Vérifions-le avec l'exemple d'Anne et de John, dont on considère qu'ils ont les mêmes préférences et les mêmes accomplissements objectifs mais qu'Anne est plus ambitieuse que John. Si ses ambitions font qu'elle se sent moins heureuse, pour un même niveau de functionings objectifs, la méthode d'indifférence traduit cette différence dans le vecteur entier des functionings, de telle sorte que la courbe d'indifférence d'Anne est plus basse que celle de John et conduit à un vecteur équivalent inférieur sur le sentier de référence. Anne apparaît alors comme étant plus mal lotie que John, non pas en raison d'ambitions plus élevées, mais $\mathrm{du}$ fait des conséquences de ses ambitions sur son propre bonheur. En effet lorsque l'on cherche à pondérer le bonheur, on est forcé de prendre en compte la satisfaction de l'individu à l'égard des résultats auxquels il est parvenu (c'est-à-dire ses accomplissements) ainsi que sa satisfaction à l'égard de ses ambitions. Pourtant, le plus souvent, on admet qu'un individu exigeant ou ambitieux, qui ne se satisfait que de résultats importants, ne devrait pas être considéré comme étant plus mal loti pour cette raison. La pondération du bonheur, dans ce cas, doit être couplée à celle des ambitions. Or la méthode d'indifférence traite deux individus, ayant les mêmes préférences mais des niveaux d'ambition très différents, comme s'ils étaient entièrement identiques. Autrement dit cette méthode permet de tenir compte des préférences, sans que les individus soient jugés entièrement responsables de leurs propres préférences. En revanche, elle leur attribue une totale responsabilité pour le niveau personnel de leurs ambitions. Il est, en effet, nécessaire, dans cette pondération du bonheur et des ambitions, d'envisager le niveau de ces dernières (c'est-à-dire leur exigence ou leur modération) et l'utilité dérivée (faible ou élevée), qui affecte les individus. Ainsi on estime que des goûts dispendieux sont généralement accompagnés d'ambitions exigeantes et se

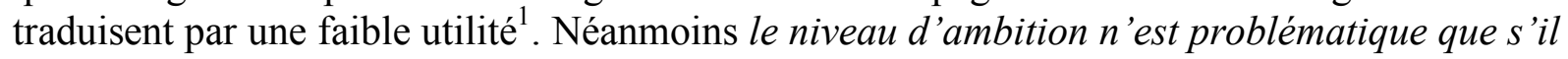
a pour contrepartie un faible niveau d'utilité. Par conséquent, lorsque les "goûts dispendieux » se traduisent dans des ambitions élevées, ils ne justifient aucune mesure de justice sociale, puisque les individus doivent assumer la responsabilité de leurs ambitions.

On peut toutefois juger que le fait de négliger totalement le niveau d'utilité et le niveau de satisfaction des agents est trop peu généreux, quand bien même une pleine attention serait accordée au contenu des préférences ${ }^{2}$. Le souci de solidarité et les devoirs, qui y sont liés, suggèrent que le fait de se sentir malheureux est une cause légitime de préoccupation sociale, quelle que soit la façon dont ce malheur est advenu. Dès lors, on peut envisager des procédés et des dispositions dont l'effet redistributif serait d'induire davantage d'égalité en matière de bonheur. Si, par conséquent, des ambitions élevées ne justifient pas, en et par elles-mêmes, d'aide sociale, les conséquences négatives de la frustration, qu'elles engendrent, peuvent

\footnotetext{
${ }^{1}$ Néanmoins les goûts d'un individu, en tant que tels, ne peuvent être jugés « dispendieux », si cet individu a de faibles ambitions et se contente d'eau et de pain, quand il ne peut pas s'offrir son festin préféré (du bordeaux antérieur au phylloxera et des œufs de pluvier). Ses goûts ne sont « dispendieux » que lorsque l'eau et le pain lui donnent une utilité inférieure à celle d'un autre individu. Ce qui est problématique dans les goûts « dispendieux », ce ne sont ni l'orientation des goûts ni ces goûts en eux-mêmes mais le niveau des ambitions.

${ }^{2}$ En effet la méthode d'indifférence satisfait les préférences de l'individu qui veut être un artiste mais néglige l'ambition de celui qui veut être un grand artiste et est malheureux de n'y pas parvenir.
} 
justifier un effort redistributif. Il s'agit là, a contrario, d'une approche très généreuse. Si l'on revient à l'exemple d'Anne et de John et que l'on considère un troisième individu, Chris, qui a les mêmes préférences qu'Anne et John, les mêmes ambitions qu'Anne mais le même niveau de functionings que John, y compris concernant le bonheur dans la mesure où, contrairement à Anne, Chris se contente de sa faible satisfaction et ne sent pas plus mal à son sujet, la méthode d'indifférence - adaptée de telle sorte qu'elle tienne compte de l'influence des ambitions des individus sur leur bonheur - traite alors John et Chris de la même façon, c'est-à-dire comme s'ils étaient entièrement identiques. Autrement dit, les niveaux d'ambitions, bien qu'ils soient différents, sont totalement négligés, lorsqu'ils n'ont aucun impact sur le bonheur. L'incidence des niveaux d'ambition sur le bonheur est prise par là en considération, quoiqu'un degré substantiel de responsabilité soit attribué aux individus, concernant leurs buts et leurs ambitions ${ }^{1}$.

Il semble donc que, par cette méthode, on puisse tenir compte, de manière appropriée, des ambitions. Bien qu'une faible satisfaction, résultant d'ambitions élevées, soit imputable à la responsabilité individuelle, les sentiments pénibles et le malheur, qui résultent du manque de satisfaction, peuvent constituer un foyer légitime d'attention sociale, ces éléments étant sans doute les seules conséquences subjectives, résultant d'ambitions élevées, qui méritent d'être prises en compte socialement. En outre, les conséquences de l'échec, consécutif à la réalisation d'ambitions élevées, concernant d'autres functionings objectifs pertinents sont, par ce biais, automatiquement prises en considération. Ainsi la méthode d'indifférence est la seule méthode permettant de tenir compte d'un déficit de bonheur affectant les individus et justifiant de compenser un individu pour le malheur dont il souffre. Le principe d'égalité des réalisations fondamentales offre donc, par une pondération différenciée des functionings retenus, une solution aux questions délicates que posait la compensation de la responsabilité en matière de bonheur individuel.

\section{Conclusion}

La méthode d'indifférence ici présentée justifie que des institutions et des politiques sociales prennent directement en compte les accomplissements sociaux des agents et, indirectement, les accomplissements des sphères privées. Elle permet de concevoir des dispositifs de redistribution initiant davantage d'égalité en matière de bonheur. Ces mesures de compensation se concentrent sur les accomplissements ou les résultats individuels, dans leurs dimensions sociales, étant admis que les individus assument la responsabilité de leurs accomplissements privés. Ainsi l'approche par les functionings actualise un souci de justice et d'équité plus large que l'approche par les chances ou par les ressources qui privilégient, quant à elles, la possibilité, pour les individus, d'être libres de choisir. Tout en tenant compte des accomplissements et de la liberté d'une façon satisfaisante, cette approche trace une sphère normativement acceptable de responsabilité personnelle. A la différence de l'attention exclusivement portée aux chances ou aux capabilités, l'approche par les functionings se soucie de la distribution des lots, une fois que les réalisations de chacun ont été menées à bien, c'est-à-dire au terme de la distribution ${ }^{2}$. Par là, elle récuse que la notion de responsabilité

\footnotetext{
${ }^{1}$ Alors que la méthode d'indifférence, comme telle, établit une pleine responsabilité individuelle en matière d'ambitions ou de niveau d'utilité.

${ }^{2}$ Le travers d'une approche par les chances ou par les capabilités est en effet de donner un poids trop grand aux chances passées aux dépens des chances et des accomplissements actuels. De même l'approche ressourciste néglige les dotations finales et l'équité de leur distribution.
} 
fournisse une justification suffisante, pour des inégalités sociales existantes ou pour la situation des pauvres jugés « non méritants » et des plus défavorisés ${ }^{1}$.

\section{Bibliographie}

Anderson, E., (1999), « What Is the Point of Equality? », Ethics, 109.

Arneson, R. J., (1989), «Equality and Equal Opportunity for Welfare », Philosophical Studies, 56, p. 77-93.

Arneson, R. J., (1990), «Liberalism, Distributive Subjectivism, and Equal Opportunity for Welfare », Philosophy and Public Affairs, 19, p. 158-194.

Arrow, K. J., Social Choice and Individual Values, New Haven et Londres, Yale University Press, 1951.

Bentham, J., Fragment sur le gouvernement, Paris, LGDJ / Montchrestien, 1998.

Cohen, G., (1989), « On the Currency of Equalitarian Justice », Ethics, 99, p. 906-944.

Cohen, G., (1990), «Equality of What ? On Welfare, Resources and Capabilities », Alternative to Welfarism (M. De Vroey éd.), Numéro spécial de Recherches économiques de Louvain, 56, p. 357-382.

Dworkin, R., Sovereign Virtue. The Theory and Practice of Equality, Cambridge (Mass.), Harvard University Press, 2000.

Fleurbaey, M., «Equality of Functionings», Mars 2004 (revu en Août 2004), http:/univpau.fr/ fleurbae/papers.htm.

Hurley, S., Justice, Luck, and Knowledge, Cambridge (Mass.), Harvard University Press, 2003.

Keller, S., (2002), « Expensive Tastes and Distributive Justice Social », Theory and Practice, 28, 4, p. 529-552.

Lippert-Rasmussen, K., (2001), « Equality, Option Luck, and Responsibility », Ethics, 111, p. 548579.

Pareto, W., Manuel d'économie politique, 1906.

Rawls, J., Theory of Justice, Cambridge (Massachusetts), Belknap Press of Harvard University Press, 1971 ; trad. franç., Théorie de la justice, Paris, Seuil, 1987.

Rawls, J., (1982), « Social Unity and Primary Goods », in A. Sen et B. Williams (éd.), Utilitarianism and Beyond, Cambridge, Cambridge University Press, p. 159-186.

Rawls, J., (1993), Political Liberalism, New York, Columbia University Press, 1993, $1^{\text {ère }}$ éd. ; trad. franç., Libéralisme politique, Paris, PUF, 2001.

Roemer, J., (1986) «Equality of Resources Implies Equality of Welfare », The Quarterly Journal of Economics, vol. 101, nº (Nov. 1986), p. 751-784.

Roemer, J., Theories of Distributive Justice, Cambridge (Mass.), Harvard University Press, 1996.

Scanlon, T., (1975), « Preference and Urgency », Journal of Philosophy, vol. 72, p. 665-669.

Scanlon, T., What we ought to each other ?, Cambridge, Harvard University Press, 1998.

Sen, A., Collective Choice and Social Welfare, Edimbourg, Oliver \& Boyd et Amsterdam, North Holland, 1970.

Sen, A., (1985a), «Well-Being, Agency and Freedom», Dewey Lectures, 1984, Journal of Philosophy, 82, p. 334-347.

Sen, A., (1985b) Commodities and Capabilities, Amsterdam, North Holland, 1985.

Sen, A., The Standard of Living, Tanner Lectures on Herman Values, Vol. VII, Cambridge, Cambridge University Press, 1986.

Sen, A., On Ethics and Economics, Oxford, Basil Blackwell, 1987.

Sen, A., " Capability and Well-Being », in The Quality of Life, 1993.

Sen, A., Development as Freedom, New York, Alfred A. Knopf, 1999.

Sen, A., Inequality Reexamined, Cambridge (Mass.), Harvard University Press, 1992 ; trad. franç., Repenser l'inégalité, Paris, Seuil, 2000.

\footnotetext{
${ }^{1}$ A trop souligner l'importance de la responsabilité dans le bien-être individuel, les institutions sociales négligent les perdants et sont trop peu généreuses à leur égard.
} 
Tinbergen, J., (1957), «Welfare Economics and Income Distribution», The American Economic Review, vol. 47, n², p. 500.

Vallentyne, P., Left Libertarianism and Its Critics : The Contemporary Debate, Londres, Palgrave, 2000.

Van Parijs, P., Real Freedom for All. What (if anything) Can Justify Capitalism?, Oxford, Oxford University Press, 1995. 